\title{
TEORÍA Y PRÁCTICA EVALUATIVA. \\ Una aproximación epistemológica
}

\section{THEORY AND EVALUATION PRACTICE. A epistemological approach}

\author{
Juan Huaylupo Alcázar*
}

\begin{abstract}
RESUMEN
Se analiza críticamente, los criterios implícitos epistemológicos en la concepción y práctica evaluativa predominante, para postular que las evaluaciones no son expresiones objetivas, universales ni técnicas. La evaluación debe ser una investigación que se aproxima a la comprensión del fenómeno evaluado y cuyo resultado será inédito en razón de las peculiaridades, del contexto histórico y coyuntural, así como, del estado del conocimiento sobre lo evaluado; incluso, de la concepción interpretativa y metodológica del investigador.
\end{abstract}

PALABRAS CLAVE: EVALUACIÓN * PODER * TEORÍA * TÉCNICAS DE EVALUACIÓN * MÉTODO EVALUATIVO

\section{ABSTRACT}

It analyzes critically, the implicit epistemological criteria in the conception and evaluative practice predominant, for postulate that the evaluations aren't objective expressions, universal or techniques. The evaluation should be an investigation which approximates understanding the phenomenon evaluated and the result will be unpublished by reason of the peculiarities, the historical and conjunctural context, also, of the state of knowledge about the evaluated; even, of interpretive and methodological conception of the researcher.

KEYWORDS: EVALUATION * POWER * THEORY * EVALUATION TECHNIQUES * EVALUATION METHODS

Escuela de Administración Pública y Sistema

de Estudios de Posgrado de la Universidad de

Costa Rica.

JUAN.HUAYLUPO@ucr.ac.cr 


\section{INTRODUCCIÓN}

La evaluación de proyectos y programas es una práctica que goza cada vez de mayor aceptación por parte de organizaciones empresariales e instituciones estatales, habiéndose convertido en las actuales circunstancias, en una actividad necesaria $y$ en un requisito para la continuidad de acciones sociales o proyectos de inversión. La importancia asignada a la evaluación, está dada por atribuírsele un carácter dictaminador sobre la pertinencia y consistencia de las actividades que se realizan en las organizaciones, ambientes físicos o naturales, tanto por entidades privadas o públicas, nacionales o internacionales.

La evaluación en las circunstancias críticas del presente globalizado, adquiere una mayor importancia ante la necesidad de conocer y corregir los impactos de acciones en espacios de intervención. Las circunstancias del devenir crítico y competitivo en la economía, la creciente iniquidad y pobreza en las sociedades o por procesos ineficientes o de dudosa idoneidad, han convertido a la evaluación en el medio útil y necesario para conocer los efectos de las acciones que se realizan en las organizaciones y sociedades. La evaluación debe implicar la generación del conocimiento de las realidades en donde se interviene o actúa para lograr propósitos definidos.

Los conocimientos y práctica evaluativa, altamente ponderados contemporáneamente, no son nuevos, forman parte de una ancestral preocupación occidental por controlar y dominar el presente y el futuro, particularmente en el sistema económico, por la necesidad de prever los resultados de las acciones 0 inversiones realizadas. Estas cualidades condicionan que la evaluación se encuentre formando parte de las prácticas administrativas de las organizaciones $y$ forma parte sustantiva en las técnicas de evaluación financiera o social de proyectos, así como, sea apreciada como inherente a los procesos de planificación. La evaluación es heredera y continuadora de tales influencias históricas y prácticas funcionales a la época, a la vez que es apreciada como una labor racional, técnica, útil y necesaria para las organizaciones. Por la importancia asignada y por realizar una tarea que es valorada como objetiva y neutral, tiene relativa autonomía de la jerarquía administrativa.

De este modo, la evaluación no solo es apreciada por la medición de la efectividad y eficiencia de los recursos o las inversiones realizadas, también se le otorga una función legitimadora de decisiones adoptadas (House y Howe, 2001).

\section{SOBRE LA CONCEPCIÓN DE LA EVALUACIÓN}

La evaluación o la valoración sobre lo actuado, deberá ser una aproximación cognoscitiva sobre las acciones y efectos de las intervenciones en las realidades, donde se esperan específicos resultados. Las acciones o alteración de las condiciones en un espacio social o natural, no necesariamente serán decisivas para el logro de los cambios intencionados, debido fundamentalmente, a la complejidad de una realidad, que no es inerte ni vacía. Estas son, según las condiciones y peculiaridades de las realidades, las que determinarán la viabilidad de las intervenciones. No se puede suponer que las acciones ajenas y externas a los ámbitos de la intervención, serán los aspectos determinantes o decisivos en el control o manejo de los resultados.

El conocimiento sobre la consistencia $y$ pertinencia de las intencionalidades de las intervenciones, es el requisito inicial a toda evaluación, así como lo es, el tener una aproximación cognoscitiva de la realidad, de la situación y las peculiaridades de la realidad donde se interviene. En estricto sentido, el objeto concreto de la evaluación deberá ser el intento de conocer la interacción dinámica de las acciones interventoras con las peculiaridades de las realidades concretas donde se actúa, lo cual brindaría la comprensión o explicación de los resultados que se obtienen. En este sentido, toda evaluación sería una investigación porque permitiría conocer la realidad donde se interviene. Sin embargo, habría que admitir que no es la forma predominante de concebir ni hacer evaluación.

De manera explícita, la evaluación es concebida como una labor proactiva, la ponderación sobre la efectividad de las acciones tiene 
como propósito fundamental el proponer cambios para incidir en el control y previsión de los resultados específicos en las organizaciones. La aceptación de la delimitación general del objeto de estudio de la evaluación, revela la vigencia del origen productivista del sistema capitalista, así como, pone en evidencia una particular concepción del mundo.

Los implícitos epistemológicos de la concepción evaluativa no se cuestionan, se comparte la creencia que toda acción humana, concebida racionalmente y con los medios adecuados, puede modelar cualquier realidad. Esta perspectiva es continuadora de una concepción $y$ práctica mecanicista, presente en las labores que son funcionales al sistema prevaleciente. Así, la omnipotencia de esta cosmovisión imagina que el mundo, las sociedades y las organizaciones, son o pueden ser, el producto intencionado de decisiones racionales y objetivas. Esta posición recoge una larga tradición teórica neoclásica, weberiana y funcionalista en las Ciencias Sociales, así como, de la epistemología $y$ metodología positivista.

La concepción del mundo centrada en los intereses individualistas y clasistas, ha modelado la actuación social de la sociedad capitalista, condicionando sus relaciones con la naturaleza $y$ el ambiente; dimensión que constituye el ámbito teórico y práctico de la evaluación, el cual no ha sido revisado ni cuestionado.

\section{LA EVALUACIÓN COMO PROPUESTA TECNOCRÁTICA}

La importancia de la evaluación podría estar asociada con la actividad investigativa, pero básica y predominantemente, está ligada con el quehacer utilitario. No se busca conocer y comprender la realidad para actuar consistente $y$ pertinentemente, lo que se propone es controlar y manipular los fenómenos para propósitos específicos, lo cual guarda semejanza con prácticas impositivas, afines en lo social, con políticas de dominación.

La evaluación o la valoración de lo evaluado, no busca conocer, busca saber los resultados de los impactos de las acciones efectuadas para ser contrastados con los resultados esperados. La valoración sugerirá acciones para cumplir o magnificar las expectativas, pues se considerará un error instrumental el no haber obtenido el control $y$ las proyecciones efectuadas. Sin embargo, la evaluación ni los evaluadores son omnisapientes, menos aún cuando se renuncia a conocer las causas de los fenómenos $y$ las condicionalidades donde se desarrollan los procesos que pretenden cambiar y controlar.

La evaluación, según Reinhard Stockmann, se diferencia radicalmente de la investigación, el utilitarismo de su quehacer ilumina su horizonte laboral, que es coincidente con las tendencias que ponderan lo pragmático y desplaza la importancia de la labor científica e investigativa.

... las evaluaciones no representan ningún fin último en sí mismas, a diferencia de lo que ocurre en la investigación científica especializada. No se desarrollan con la finalidad exclusiva de obtener conocimiento, sino que deben ser de utilidad $y$ contribuir a hacer los procesos transparentes con la finalidad de facilitar la toma de decisiones. Por ejemplo, con el objetivo de conformar en forma más efectiva los procesos de desarrollo, manejar los recursos más eficientemente, aumentar los productos o servicios, mejorar el grado de impacto, asegurar la sostenibilidad etc., las evaluaciones pueden - tal como los sistemas de administración de calidad-contribuir a mejorar la calidad de un programa o un servicio (Stockmann, 2009: 68).

En su forma más radical, la evaluación se transforma en una combinación constructivista de negociaciones, desarrollo organizacional y terapia de grupo, que en ninguna forma busca explicaciones científicas superiores, sino la emancipación $y$ el empoderamiento de los stakeholders reprimidos (Stockmann, 2009: 77).

La evaluación como conocimiento que cree saber cómo obtener resultados deseados, se distancia del quehacer científico, al asumir un pensamiento mágico que cree viable la 
predicción. Así, Reinhard Stockmann se presenta, entre otros, como un sustentante que imagina un mundo pautado donde es posible erradicar la incertidumbre.

Evaluar es discernir, sopesar criterios, aplicar el razonamiento para identificar las causas de los problemas en la búsqueda de su mejoramiento (Picado, 2002: 9).

La evaluación es el proceso de juzgar el mérito de algo y, específicamente, en el área social busca garantizar un mejor cumplimiento de los programas y proyectos (Picado, 2002a: 47).

La evaluación es un proceso que compara lo observado con lo estándar o esperado. Dos ideas sobresalen en esta definición: la idea de observación requiere que decidamos cómo se relaciona lo observado con lo estándar o esperado. Para comparar hay previamente que conocer lo estándar (Amezcua, 1996: 2).

... la evaluación es una herramienta fundamental para iluminar la toma de decisiones en las diferentes fases de ejecución de los programas e intervenciones, desde su concepción y diseño hasta su posterior valoración en términos de logros e impactos (Montero, 2004: 2).

La evaluación de un programa o proyecto, supone que las acciones aplicadas son las que han originado los cambios ocurridos en la realidad, luego se asume que modificando las acciones es posible modelar racionalmente la realidad. Desde esta perspectiva, los fenómenos son de determinada forma y naturaleza, según las intervenciones que actúan sobre ellos, sin ninguna peculiaridad y comportamiento que le sea propio. Así, la realidad, objeto de la intervención evaluativa, no tiene ninguna alternativa de reacción que le sea peculiar, imagina que las realidades se comportarán de manera idénticas ante las iguales acciones, lo cual es una posición arcaica, estereotipada y mecanicista.

El devenir de las organizaciones condicionado por patrones sociales, políticos y culturales, así como, por las condiciones cotidianas $y$ por su naturaleza peculiar, es inexistente en la concepción evaluativa que cree que solo las intervenciones adecuadas dan resultados previsibles. La adopción de esta cosmovisión es totalmente contraria a lo que sostiene Niklas Luhann (1998), al apreciar a la sociedad como un sistema autorreferencial y autopoiético, que se reproduce a sí mismo en una red de operaciones que le son propias e independientes de las estructuras y del sistema, lo cual es un conocimiento más complejo, que la simple creencia de un mundo hecho a imagen de la acción racional. Un conocimiento discrepante con esta visión es el concebir a la sociedad $y$ a la naturaleza como sistemas abiertos, cuyas dinámicas son particulares e inéditas en relación con sus propios sistemas en interacción con otros, donde lo propio $y$ lo ajeno se confunden $y$ se transforman inéditamente. Los fenómenos transcurren de modo inesperado a nuestras creencias, conocimientos e ignorancia sobre la complejidad de lo real, sin embargo estos criterios, que relativizan el dominio de la acción intencionada, están ajenos a la teoría y práctica evaluativa.

... la idea de que la aparición de contradicciones $y$ antinomias no señala las profundidades de lo real. Éstas se revelan donde nuestros instrumentos teóricos $y$ lógicos fracasan, donde nuestra lógica enloquece y se paraliza. El conocimiento del conocimiento nos enseña que sólo conocemos una delgada película de la realidad. La única realidad que nos es conocible es coproducida por el espíritu humano, con la ayuda de la imaginación. Lo real y lo imaginario están cotejidos y forman el complexus de nuestros seres $y$ nuestras vidas. La realidad humana en sí misma es semi-imaginaria. La realidad es solamente humana y sólo parcialmente es real (Morín, 1995: 10).

La historia de la humanidad y particularmente en Occidente, como también lo destaca 
Weber (1977), muestra una creencia recurrente: tener el dominio sobre el presente y el futuro. En esa perspectiva, el pasado no importa, porque supuestamente es inexistente $y$ sin capacidad de determinación alguna. Así, la magia, la religión o los mitos, finalistas o fatalistas, sin realidad ni historia, han acompañado las especulaciones sobre el futuro de los pueblos $y$ organizaciones. En la era moderna, por el prestigio de la ciencia, se le confirió la facultad de predecir. Mario Bunge $(1997)^{1}$, que entre otros lo divulgan como una característica de la ciencia y del quehacer académico cotidiano donde predomina la epistemología positivista.

Esta situación especial de conocer cómo se conoce resulta tradicionalmente elusiva para nuestra cultura occidental centrada en la acción y no en la reflexión, de modo que nuestra vida personal es, en general, ciega a sí misma. En alguna parte pareciera hacer un tabú: "Prohibido conocer el conocer". Pero en verdad el no saber cómo se constituye nuestro mundo de experiencias, que es de hecho lo más cercano de nuestra existencia, es un escándalo. Hay muchos escándalos en el mundo, pero esta ignorancia es de los peores (Maturana y Varela, 1999: 19).

De manera específica y concreta, siendo la evaluación efectuada en organizaciones, su propuesta se centra en una determinada actuación administrativa para la optimización $y$ el uso de los recursos, con el fin de hacer

1 La obra de Mario Bunge - formador de generaciones de profesionales en América Latina- no ha rectificado esa idea, a pesar de su demostración en contrario. En tal sentido, comparte pensamiento con Milton Friedman y futurólogos del pasado y el presente. Consideración que incide directamente en la definición de ciencia, que en lugar de apreciarla como un conocimiento aproximativo de la realidad, que busca comprender y explicar los fenómenos existentes, cree que es posible determinar el futuro a partir de conocimientos parciales $y$ parcializados $y$ bajo supuestos mecanicistas, al mejor estilo newtoniano. La ciencia no puede estudiar el futuro, porque solo existe en la ilusión, las creencias o la especulación. eficientes y eficaces a las organizaciones. En tal sentido, la postulación no es original, ha sido una preocupación y acción administrativa inspirada en la productividad empresarial, la cual tiene como exponentes a los ingenieros y propietarios, Robert Owen (1771-1858), Charles Babbage (1792-1871), Henry Metcalfe (1805-1881) y Roswell Lee (1829-1833), entre otros que fueron precursores de las ideas de Frederick Taylor (1856-1915) y Henry Fayol (1841-1925), a quienes se les recuerda como fundadores de la escuela clásica o del mecanicismo en la administración (Neffa, 1990), aún cuando algunas de sus ideas habían sido enunciadas, 84 años antes de la obra de Taylor de 1916.

La impronta omnipotente del sistema prevaleciente, tiene en la dimensión técnica el fundamento que justifica su actuación. La técnica y no el conocimiento científico, es la base del quehacer pragmático y utilitarista del capitalismo, aún cuando ha sido falsificada como la materialización u objetivación de la ciencia, quizás por ello la indiferenciación, entre ciencia y técnica, que se establece en determinados círculos. La racionalidad para hacer cualquier cosa, magnificó a la técnica e imaginó un mundo mecánico, que cautivó a Descartes, Newton, Arquímedes, Taylor, entre otros muchos destacados personajes del pasado $y$ del presente, que creían que la técnica era equivalente a la ciencia. La configuración técnica de la ciencia, data del siglo XVII y tiene cada vez más seguidores, incluso en los ámbitos académicos.

La ponderación en favor de la evaluación está asociada con la atribución asignada a la racionalidad y capacidad de la transformación humana, facultades del homo sapiens $y$ del homo faber, no pueden ser negadas; sin embargo, su capacidad de actuación es plural y diversa en su historicidad e identidad, así como, en sus situaciones y condiciones de acción. La omnipotencia de la acción colectiva no es absoluta, como tampoco lo es, la racionalidad ni la razón instrumental.

La magnificación de la razón paradigmática o técnica, es presentada como omnipotente, que encubre el dominio de intereses 
que se impone a la pluralidad de alternativas de actuación. La negación de la diversidad de acción en la heterogeneidad social y orgánica, es una posición de dominio frente a otros, que está implícita y compromete a toda evaluación, como trabajo privado. La evaluación reedita el privilegio de la razón instrumental (Horkheimer, 1973) o del cómo hacer las cosas frente a la generación de conocimiento que permita comprender la realidad. No es posible imaginar individuos, grupos o sociedades que puedan convertir a los otros, a la naturaleza o al ambiente, en cosas inertes. La conversión de los sujetos en objetos, es una relación política de dominación y está implícita en la sobreponderación de las técnicas, aún cuando, las realidades ni las relaciones sociales son moldeables, como la arcilla en manos del alfarero.

Al mecanicismo en el pensamiento $y$ práctica administrativa, vigente en las organizaciones, se le agregan sucesivas técnicas, como las de justo a tiempo, círculos de calidad, reingeniería, bechmarking, outsourcing, downsizing, rightsizing, la nueva gerencia pública, etc., como posiblemente le seguirán otras que se mercadean, como poseedoras de ilusorias capacidades para dominar y controlar el uso de los recursos y sus resultados en las organizaciones $^{2}$.

Creer que las sociedades y el mundo podrán cambiar en determinado sentido $y$ orientación, a razón de acciones ajenas a ellas, como pensaban Arquímedes (287-214 a.C.) o Comte (1798-1857), son ideas simples en un imaginario mundo mecánico. El concebir que acciones que se imponen a las organizaciones y sociedades, para lograr y perennizar propósitos particulares, no será racional ni objetivo, es un proceso político de dominación, que trasciende lo administrativo y lo evaluativo.

$2 \quad$ El pensamiento $y$ práctica mecanicista no son ni han sido exclusivas de la administración, forman parte del pasado de muchas de las ciencias. Así por ejemplo, en América Latina se afirmaba que el modelo de desarrollo pensado por la CEPAL, era el causante de la crisis ocurrida en la década del ochenta, por ello se implementó un nuevo modelo, los programas de ajustes estructurales a los que han seguido los tratados de libre comercio y la desregulación y privatización del quehacer estatal.
En nuestros días, los discursos administrativo, empresarial y evaluativo, coinciden en creer que las prácticas arraigadas históricamente en las organizaciones y sociedades, son un obstáculo para la innovación y la competitividad, por ello expresan la necesidad de modificar las culturas a razón de intereses empresariales, gubernamentales o de algún autócrata iluminado, lo cual es una posición voluntarista o individualista metodológico. Las culturas son identidades sociales, construidas colectiva e históricamente por los pueblos y sociedades. El reconocimiento de la interdependencia en sociedades desiguales, así como, su complejidad, cuestiona la efectividad de dogmáticas certidumbres (Prigogine, 1996).

La aplicación de procesos estandarizados y mecánicos en un mundo que no lo es, obtendrá resultados distintos, en razón de la peculiaridad de cada realidad. Las múltiples evidencias de un mundo que no responde de manera estandarizada, que es diverso y plural, no ha sido suficiente para erradicar esa visión simplificada y absolutizada de la realidad. El mecanicismo es una ideología sin sustento real, que justifica dogmatismos que afectan las relaciones entre actores sociales, la sociedad, el Estado y el ambiente.

Los fenómenos no se repiten de manera idéntica ni son estáticos, así como, tampoco se transforman estandarizadamente, ni se comportan según patrones teóricos, tendencias estadísticas, sino por la naturaleza y peculiaridad de los fenómenos en sus contextos. De este modo, la evaluación no puede ser concebida como un conocimiento que pueda predecir, como tampoco es una técnica probabilística. Las técnicas evaluativas miden $y$ describen parcialmente una realidad, de ninguna manera, explican ni permiten comprender los resultados obtenidos, porque sus significados están arraigados al contexto y a la realidad evaluada. Iguales resultados en distintas realidades o fenómenos, tienen diferentes significaciones, ni pueden ser interpretados de manera similar con "teorías" formalistas, como tampoco comparados.

La complejidad de la realidad y relatividad del conocimiento científico, no son ficciones. La simplificación e ignorancia de la 
complejidad de los problemas sociales, del desarrollo o los éxitos empresariales, ha fetichizado al dinero, como omnipotente para todo, aún cuando su dilema y paradoja, es hacerlo con menos dinero.

El fin de las creencias de un mundo mecánico que es posible dominar y controlar, así como, suponer que los conocimientos de esa realidad puedan ser exactos, absolutos y universales (Prigogine, 1996), aún no es aceptado, las concepciones omnipotentes y omnisapientes, se resisten a renunciar a la posibilidad de dominar y controlar la acción humana y sus resultados. No es la carencia de estudios e interpretaciones complejas lo que posibilita la adopción de prácticas simplificadas, son los intereses y poderes autoritarios los que imponen fantasías absurdas, en los espacios de su dominación y propiedad.

Las necesidades y problemáticas de un mundo en crisis, se magnifican ante intervenciones que responden a cosmovisiones simplificadoras y erróneas de las realidades. Las viejas soluciones para nuevos problemas, perenniza ideas, justifica relaciones desiguales y agudiza la crisis. Estas consideraciones no son ajenas a la evaluación, forman parte constitutiva de su quehacer, precisamente en momentos de crisis económica y de ilegitimidad social del Estado.

El conocer el origen de los fenómenos, sus causas y sus efectos, ha sido el objeto de la ciencia, así como, de sus logros y su prestigio a lo largo de los tiempos. El quehacer científico ha avanzado por la imaginación, para interpretar el mundo real, pero no sin la oposición de las creencias, tradiciones, intereses y poderes en todas las épocas. La renovación permanente del conocimiento científico, relativiza los conocimientos validados de una época, así como, afecta a quienes el conocimiento anterior les es útil y funcional. Los conocimientos son productos históricos, su pertinencia social no cambia automáticamente cuando cambian los conocimientos, porque están arraigados a tradiciones, prácticas, intereses y poderes. Los científicos no tienen la capacidad para sustituir argumentos y creencias de las sociedades y las épocas, así tampoco, límites para incidir en los contextos científicos o académicos, porque los conocimientos están arraigados a formas sociales que trascienden al razonamiento y demostración científica. Así, los conocimientos que son funcionales al poder, gozan de una extraordinaria difusión, promoción e incentivos que contribuyen a reafirmar $y$ fortalecer las creencias y prácticas prevalecientes, mientras que aquellos que revelan las causas de la desigualdad e iniquidad, así como, las tareas para el bienestar social o la emergencia de una nueva conciencia ecológica, no tienen la fuerza política para sustituir o liquidar las prácticas que degradan el medio ambiente o atentan contra el bienestar social y los derechos humanos. La viabilidad social de aceptación a las nuevas explicaciones está relacionada con las variaciones contextuales y de la época, los cambios cognoscitivos no cambian culturas ni sociedades. Los descubrimientos $y$ conocimientos acumulados en la Biblioteca de Alejandría, habrían hecho progresar a la humanidad en mil años, quizás ello, fue la causa de su destrucción.

La pretensión de conocer el futuro se ha metamorfoseado con ropajes distintos a través de los tiempos. En los primeros momentos, las creencias mesiánicas dictaron el futuro $y$ actualmente, se le asigna a la ciencia, las teorías, las técnicas, las estadísticas o la informática, la capacidad para fundar predicciones que justifican acciones de poderes arbitrarios en las organizaciones empresariales y estados privatizadores.

La compresión de los fenómenos sociales, como los procesos evaluativos, requieren ser analizados en su complejidad e interdependencia, no como unidades autodeterminadas ni libres de los condicionamientos del pasado y del presente. La unidad inextricable de fenómenos en apariencia desarticulados, debe ser el fundamento de la labor evaluativa. No será evaluación una valoración social, medida, comparada o interpretada con criterios ajenos al fenómeno $y$ las circunstancias históricas y coyunturales de su devenir. Asimismo, las condiciones ambientales y físicas, donde se desenvuelven las relaciones sociales deberán ser objeto de análisis, porque son parte de la conformación de tejidos sociales que condicionan resultados 
particulares. No existe fenómeno social que sea autopoiético ni producto de voluntades autocráticas.

\section{LA APARENTE NEUTRALIDAD VALORATIVA EN EL PENSAMIENTO EVALUATIVO}

La pretensión de neutralidad y objetividad de la labor del evaluador es una calificación que pretende crear la ilusión de un trabajo sin condicionamiento valorativo ni ideológico, así como, sin preferencias analíticas, según la formación profesional o experiencias de los evaluadores, lo cual es una falsificación de la naturaleza humana, que busca una apriorística validación de la evaluación. Las ideas de una práctica humana que se autodetermina, recuerda las críticas de Alvin Gouldner (1979) a Max Weber, pues el simbólico antiminotauro criticado, aún muestra tener la vitalidad de un dios pagano, que ofrece verdades sagradas encarnadas en humanos. Lo sagrado no requiere demostración, es verdad absoluta, basta su enunciación para estar por encima de mortales $y$ de cualquier argumento que lo relativice. Es de dioses imaginarios el estar libre de toda valoración, de todo compromiso, de toda influencia humana y social. Sin duda, sostener la neutralidad valorativa es una posición dogmática, es un pensamiento único que impone interpretaciones y prácticas unilaterales, así como, es una racionalidad imaginada como perfecta, objetiva, universal y exacta, que va más allá de todo quehacer científico y humano.

El objetivismo y la neutralidad, asociados con la racionalidad, que postulaba Max Weber (2001), es también un pensamiento recurrente en el pensamiento de Stockmann (2009) y de la tradición idealista alemana, lo cual ha tenido una amplia aceptación desde Talcott Parsons (1968) hasta nuestros días con las prácticas empiristas, que paradójicamente concuerdan, con Spinoza y Descartes - desde la perspectiva de Heidegger - “... en la determinación del ser como cosa, o sea, en la preparación del mecanicismo y naturalismo moderno" (García, 2003: 196) y como tal, posible de ser desarraigada de toda subjetividad que contamine las interpretaciones. Esta posición podría ser denominada como un objetivismo idealista, o paradójica- mente, como un materialismo metafísico “... que cae en una forma barroca de idealismo platónico..." (Gramsci, 1986: 291), lo cual es una contradicción que concibe fenómenos y hechos sin procesos sociales, sin historia ni sociedad.

La ponderación de la técnica o de la aplicación mecánica de procedimientos es el medio a través del cual, mágicamente se elimina toda decisión valorativa en el quehacer evaluativo y administrativo. Así, se cree erróneamente que la técnica elimina toda distorsión subjetiva, para obtener resultados tangibles de validez intersubjetiva, pues se cree empiristamente que todos reconocerán y llegarán a los mismos resultados aplicando las mismas técnicas. De este modo, la técnica es ideologizada e incontrovertible tautológicamente, aún cuando las técnicas han sido creadas $y$ diseñadas para ser empleadas de determinado modo $y$ en condiciones particulares, como una manera para lograr propósitos específicos. Hay valoración subjetiva al usar determinadas técnicas, así como la hay, al interpretar los resultados.

La pertinencia de la técnica no está dada en sí misma, tiene un fundamento cognoscitivo que le dio origen, responde a condiciones del contexto y de las necesidades concretas, su funcionalidad no abstracta es concreta e ideológica (Habermas, 1984). Las técnicas están impregnadas del espíritu y necesidades de la época, de ninguna manera son creaciones arbitrarias, así como, tampoco son producidas, mercantilizadas ni usadas de manera casual, además, no son neutrales ni objetivas. Asimismo, la utilización de la técnica requiere condiciones para su funcionamiento, las cuales no son múltiples ni indeterminadas, como tampoco se podrá obtener un resultado para el cual no fue creada.

La magnificación de la técnica ignora que existen muchas formas de hacer las cosas, esa universalización no distingue particulares en las realidades ni en las técnicas. En este sentido, la técnica es también un medio para justificar, supeditar o alienar el comportamiento humano en torno a las prácticas efectuadas desde el poder, así como, es una vana pretensión de negar subjetividad a la obra y acción humana.

Esta concepción y práctica están regularmente presentes en las evaluaciones e 
investigaciones cuantitativas, así como, en las fantasías de algunos que buscan indicadores predictivos (Montero, 2001) y otros que creen haberlos encontrado (Paula, 1992), por ello otorgan una significación que los indicadores no poseen: el saber anticipadamente el desenvolvimiento de las personas, organizaciones o economía, independientemente de cualquier circunstancia individual, social, coyuntural o histórica.

La racionalidad en Stockmann es única, solo admite una racionalidad fundada en la autosuficiencia de quienes realizan las acciones, a pesar de las distintas racionalidades producto de las diferentes clases sociales, grupos de interés o de individuos con perspectivas analíticas particulares. Esa racionalidad asociada con el pensamiento único, es también el marco de la labor evaluativa, que es compartida con otros:

Razonar bien tiene que ver con las razones que ofrecemos a favor o en apoyo de una creencia, de una acción, o de una respuesta. Se razona bien, ...cuando las razones ofrecidas son las razones pertinentes $y$ apropiadas para aquello con relación a lo cual se presentan. [...] Se trata entonces de que las razones aducidas hagan más probable que la creencia sea verdadera, que la acción sea correcta, o que la respuesta sea apropiada. La búsqueda de criterios, de normas, que permitan juzgar cuándo las razones esgrimidas realmente dan apoyo, cuándo dan fundamento, a una determinada solución, es tan decisiva como difícil (Picado y Ramírez, 1989: 14).

Para la conceptualización y ejecución de una investigación es importante qué paradigma de investigación se use. A grandes rasgos, se puede diferenciar entre dos enfoques principales. Los unos consideran la evaluación como un procedimiento empírico-científico, que sigue una lógica investigativa racional-crítica $y$, en principio, considera aplicables todos los métodos empíricos de investigación. De esta forma, la evaluación se debe enten- der como investigación social aplicada, la cual debe tomar en cuenta ciertas condiciones especiales de investigación y tiene un interés específico de conocimiento $y$ aprovechamiento, en el que se encuentra en primer lugar la utilización de los resultados de la evaluación para la "práctica" (Stockmann, 2009: 76-77).

Los criterios de evaluación deben derivarse, esencialmente, de la naturaleza del producto y de la función para la que haya sido diseñado (House y Howe, 2001: 47).

La racionalidad enunciada en la evaluación es transferida o concretada al paradigma explicativo, a la teoría adoptada o a los propósitos que se buscan conseguir. Así, la realidad no será evaluada en razón de sus peculiaridades ni de sus protagonistas sociales, sino en razón según la teoría usada. Esta posición confirma que la realidad evaluada no tiene un estatuto propio ni tiene capacidad determinadora alguna sobre su propio devenir. Tampoco su unidad o totalidad orgánica, interviene en su acontecer, pues, será la teoría o las variables e indicadores que la teoría privilegia, las que deberán ser observadas, medidas e interpretadas. Esta forma de concebir y realizar la evaluación, es la concreción de una epistemología positivista formalista que falsifica la realidad (Castells y de Ipola, 1983).

La evaluación predominantemente de Stockmann y otros, es inconsistente al concebir que lo evaluado está comprendido en la teoría o que existen conocimientos que pueden ser aplicados en razón de lo que se quiere obtener, lo cual contradice la supuesta neutralidad, pero además se asume que las aplicaciones instrumentales garantizan los resultados deseados. El uso de las teorías o paradigmas, como un mercado abierto, es una ocurrencia que desvirtúa la concepción y función de la teoría ${ }^{3}$, convirtiéndola en técnicas. Así, la

3 En sentido estricto, la teoría es la explicación comprobada de una realidad específica, o dicho de modo distinto, la exploración de la realidad a través de la investigación crea teoría, la cual no es transhistórica ni universal, luego, no es posible suponer que las explicaciones concretas puedan ser usadas 
evaluación se reduce a la aplicación mecánica de teorías, indicadores, instrumentos y relaciones estadísticas.

En la perspectiva evaluativa que se analiza, es posible inferir que siendo toda acción colectiva susceptible de ser evaluada, en razón de las racionalidades que las promueven, ellas podrían ser valoradas bajo el prisma de teorías o modelos determinados. Esto es, definir la evaluación como "una investigación social aplicada" (Stockmann, 2009:77), supone la adopción de algún paradigma aplicable al caso evaluado, consideración que imagina la existencia de teorías para cualquier caso evaluado y con independencia de contexto social y temporal. Así, la evaluación sería un macro o meta conocimiento, que superaría a las ciencias, porque sabe cómo modificar cualquier realidad. En esta perspectiva, no hay necesidad de crear paradigmas explicativos, ahí están, solo hay que aplicarlos como recetarios. El estado del conocimiento epistemológico y científico que estima que cada realidad es peculiar e inédita y que por lo tanto, tiene una explicación que es particular según su naturaleza y relaciones específicas en cada tiempo-espacio, es sustituido regresivamente por un pensamiento único y universal.

Reafirmando el principio de que en evaluación se debe dar preeminencia al análisis y al discernimiento, busca asignar al análisis del problema al cual responde el programa o proyecto evaluado, el punto de partida para el razonamiento evaluativo (Picado y Ramírez, 1989: 13).

La teoría es la asimilación crítica del conocimiento del pasado y del presente, que contribuye a explicar la realidad estudiada, a través de ella, se intenta reproducir comprensivamente los fenómenos, de ninguna manera regulan las realidades. Los conocimientos científicos no

como técnicas. Las teorías son productos históricos, creadas en condiciones particulares de los fenómenos que explican. Asumir que alguna teoría debe ser aceptada $y$ aplicada, por el reconocimiento intersubjetivo existente, es una validación tautológica, sin relación con lo evaluado. Pretender liberar a las teorías del tiempo-espacio las deja vacías y las convierte en dogmas ideológicos. pueden independizarse del fenómeno estudiado, su pertinencia no tiene una determinación tautológica, ni su validez puede ser definida exclusivamente por comparación con otras teorías, como algunos adoptan (Escudero, 2006).

Los argumentos por la racionalidad, objetividad y neutralidad valorativa en la evaluación, son solo aparentes. Stockmann y otros reconocen similarmente que:

La valoración de los hechos evaluados no se orienta, sin embargo, según normas preestablecidas (como en el caso de ISO -International Organization for Standarization-) o parámetros (como en el EFQM -European Foundation For Quality Management-), sino según criterios que pueden ser de muy diversa índole. Estos se orientan, muy a menudo, de acuerdo con la utilidad intencionada, un hecho o un proceso de desarrollo para ciertas personas o grupos. Los criterios de evaluación pueden ser determinados por el comité de evaluación, el grupo meta, los grupos de interés (stakeholders), el mismo evaluador o por todos ellos en conjunto. Es evidente que según la escogencia de los criterios, la valoración de la utilidad efectuada durante una evaluación puede tener resultados muy diversos (Stockmann, 2009: 68).

Para que la evaluación sea útil, ha de seleccionar normas de valoración adaptadas a los gustos y necesidades de la interesada (House, 1994: 18-19).

Evaluar implica establecer un juicio sobre el valor o mérito de algo, una conclusión valorativa. Para ello se requiere siempre la comparación con algún estándar o punto de referencia que ha sido establecido explícita o implícitamente (Montero, 2004: 2).

Los valores son escogidos sin más por las personas y no están sujetos a una deter- 
minación racional. Por eso, los evaluadores deben extraer conclusiones basadas en los valores de los interesados y los clientes, sin criticar esos valores, o deben calificar marcadamente las conclusiones de los estudios de manera que se correspondan con los valores de los clientes o los interesados (House y Howe, 2001: 18).

Toda evaluación parte del interés por solucionar o encontrar respuesta a un problema. En la medida en que este haya sido definido claramente, la evaluación se orientará con mayor facilidad. Su definición debe reflejar los intereses de sus demandantes (Picado, 2002a: 54).

Esta consideración relativiza la posición aséptica que se pregona en la labor evaluativa. La neutralidad y objetividad es retórica, pues es una posición interesada desde el momento mismo de definir lo que se evalúa y cómo se evalúa, así como, en las propuestas de cambio sobre lo evaluado. La subordinación de la actividad evaluativa es transparente, llegando a ser justificadora de apreciaciones y decisiones de la gestión organizacional. El formalismo o la preeminencia de la teoría en la evaluación, se transforma en un quehacer epistemológico del individualismo metodológico, al evaluar según los criterios de los clientes, lo cual hace inconsistente el quehacer evaluativo. La evaluación es importante, pero no precisamente por los vaivenes positivistas de sus interpretaciones, sino porque toda evaluación debe ser una investigación con compromiso ético.

El conocer la realidad ha contribuido en hacer menos imprevisible el futuro, pero de ninguna manera es posible la predicción, ni ella tiene relación con el uso extensivo de las estadísticas, así como, no explican, tan solo describen parcialmente. Las cuantificaciones tan usadas por el empirismo, también muy empleadas por las evaluaciones, actúan como elemento justificador de la neutralidad valorativa porque se asume objetividad, ya que se usa extensivamente la estadística y sus modelos. El ignorar la significación valorativa o interpretativa de las cantidades, es imaginar absurdamente un mundo de cosas y no de humanos. Las cantidades que buscan representar realidades tienen significaciones que trascienden a sus magnitudes, están relacionadas con la naturaleza de lo que se mide; con las explicaciones adoptadas respecto fenómeno medido, de la forma como se han construido las cantidades, del contexto que incide en las magnitudes $y$ de los usos que se dan a los números. Las cantidades no tienen significación explicativa ni evaluativa por sî mismas (Huaylupo, 2008).

En las prácticas evaluativas y en las técnicas de proyectos, se emplea constantemente las probabilidades como criterio para prever resultados futuros, lo que en sentido estricto es suponer que las realidades futuras se comportarán según las tendencias cuantitativas de una serie histórica de datos, si fuera cierta tal especulación contraria a la historia, sociedades $y$ universo, los seres humanos no habríamos salido aún de las cavernas. La probabilidad de ocurrencia de algún acontecimiento no es un asunto estadístico, es inherente a la naturaleza, peculiaridad y dinamismo del fenómeno (Cohen, 1952) ${ }^{4}$. El desconocimiento de las determinaciones de los fenómenos no puede ser sustituido con una serie histórica de datos y menos aún, anticipar su comportamiento. Las cantidades y sus proyecciones carecerán de toda relación con la realidad. El conocimiento de las determinaciones causales de los fenómenos podrá estar acompañado de correlaciones estadísticas (Cohen, 1952), pero de ninguna manera en sentido inverso.

La significación de las cantidades que representan realidades, está relacionada con el conocimiento sobre lo medido, mientras que las cantidades sin referencia cognoscitiva sobre la realidad, no la representan ni producen conocimiento, así como, carecen la viabilidad para transformarlas o predecir su comportamiento.

La creencia de un mundo regido por las matemáticas, es un prejuicio de la sociedad capitalista que usa y le es funcional, pues con ella, se han construido modelos útiles a los

$4 \quad$ Raphael Cohen Morris afirma en razón de las probabilidades que: "se ha hecho popular decir que la probabilidad expresa sencillamente nuestra ignorancia" (1952: 189). 
intereses de los propietarios. El atribuir a la matemática ser el lenguaje de la ciencia, reduce y simplifica las explicaciones de las realidades, así como, desconoce u oculta ser un invento y una convención humana el aceptarla. Los conocimientos plasmados por la imaginación humana, como lo es la matemática, no tiene la capacidad para representar la realidad. La paranoia de construcción de índices y estadísticas, sin referencia cognoscitiva de lo medido, es un desperdicio y una falsificación de la realidad. La evaluación y la técnica de proyectos de inversión o sociales, está colmada de trabajos cuantitativos que podrán ser mercadeados en un mundo de consumidores ignorantes.

La magnificación de los datos como equivalentes de realidad y objetividad, tiene en el filósofo griego Epicteto (55-135 d.C.), a uno de sus primeros sustentantes, quien afirmaba: "lo que turba a las personas no son las cosas sino las opiniones que de ellas se hacen" (2003: 6). La objetividad más allá de la conciencia y percepción de los individuos, sin duda no es de humanos, pero la sobreponderación a los datos no termina ahí, se cree que las nuevas cifras producto de correlaciones estadísticas, conforman nuevos datos aún cuando no corresponda con ninguna realidad empírica, ni sea expresión de alguna consideración hipotética o analítica. La autonomización de los datos en los cálculos probabilísticos, como en las interrelaciones o correlaciones estadísticas (Backhoff, Tirado y Larrazolo, 2001), son especulaciones y contradicciones empiristas, donde las nuevas cifras creadas solo tienen una existencia ficticia. Las magnitudes sin realidad no son datos y sus relaciones cuantitativas son juegos especulativos.

Los datos tienen contenidos teóricos porque han sido construidos de modo determinado, según las perspectivas interpretativas del investigador sobre el objeto, de la naturaleza $y$ la peculiaridad del objeto, del estado del conocimiento sobre el objeto $y$ del contexto donde se registra el dato. Luego, los datos tendrán significaciones particulares y poseerán implícitos contenidos teóricos; sin embargo, ello no implica que los datos tengan exclusiva y única significación, pues un mismo dato podrá tener distintas significaciones al ser articulado en discursos y análisis diferenciales (Carr, 1978; Zemelman, 1993). De esta manera, los datos llevan el sello de su construcción originaria y de su relatividad.

La creencia de que los datos son inobjetables, de objetividad absoluta, es una vana suposición empirista. La significación de los datos está en correspondencia con las variables que representan la aproximación cognoscitiva del fenómeno estudiado. Los datos señalan, muestran, designan o son indicadores de variables, según atributos dados por las creencias o teorías. Los datos son registros parciales y relativos de la realidad (Huaylupo, 2008), su significación y su relatividad están en relación directa con la forma como han sido construidos, el contexto en el cual se han registrado y de los usos particulares en las perspectivas donde son empleados. No hay datos independientes o sin implícitos interpretativos, son parte de quien lo crea, construye y usa.

La magnificación de las cantidades ha llevado a suponer la existencia de indicadores predictivos. Así se cree, por ejemplo, que es posible prever y conocer la vocación profesional y rendimiento académico de los estudiantes, de acuerdo a las respuestas a un cuestionario, construido a partir de la coincidencia cuantitativa en los indicadores, aplicados a los estudiantes al inicio y cuatro años después. La validación de esos indicadores es efectuada según los rendimientos académicos obtenidos por los estudiantes. De ese modo, no habría necesidad de explorar rendimientos académicos de los futuros estudiantes para ser admitidos a una carrera profesional, bastaría saber las magnitudes en los indicadores aplicados para aceptar o rechazar estudiantes a una carrera profesional (Montero; Villalobos y Valverde, 2007).

Los juegos experimentales que buscan coincidencias cuantitativas de indicadores, con independencia cognoscitiva de los estudiantes y su contexto, así como, de las circunstancias y vicisitudes transcurridas en el tiempo, no tendrían mayor importancia, pero al convertirse en un instrumento adicional para la exclusión a carrera de estudiantes que aprobaron el ingreso a una universidad pública en Costa Rica, sin duda es preocupante $y$ negativo. Esos indica- 
dores revelarían para esos sacerdotes del idealismo simbólico de las cifras, los rendimientos satisfactorios y la calidad de los profesionales, independientemente de la calidad $y$ pertinencia de los estudios, de la realización de las potencialidades y capacidades de los estudiantes, de los cambios en las profesiones, etc., lo cual es un atentado a la inteligencia y al quehacer investigativo y científico. Ejemplos similares $y$ dramáticos ocurren en las previsiones sobre la salud pública, la seguridad y soberanía alimentaria o el desarrollo nacional. Los cuantificadores creen haber descubierto la piedra Rosetta que les permite saber el futuro. El sacerdocio o la alienación cuantitativa y dogmática, se encubre de aparente racionalidad y cientificidad, para revelar ficciones $y$ artificios cuantitativos absurdos (Garcia, 2006: 115).

La convicción o creencia que es posible controlar los resultados de las acciones humanas, parte de suponer que la racionalidad y la manipulación de las técnicas sobre los objetos, pueden lograr cualquier resultado. Esa ingenuidad o engaño intencional es la reproducción del mecanicismo que exige obediencia sin reflexión ni crítica. La pretensión modeladora de la realidad, como el diseño y ajuste a una máquina, es una concepción antidemocrática.

\section{EL MÉTODO EN LA EVALUACIÓN}

Al método empleado en el proceso evaluativo se le ha otorgado una importancia decisiva, en la calidad y pertinencia del trabajo realizado, de modo similar al que se le ha dado por más de un siglo al método científico en la epistemología empirista.

Una de las mayores preocupaciones en el campo de la evaluación es poder mostrar la solidez o fundamentación de las conclusiones. En gran medida esta tarea se ha apoyado en el instrumental metodológico empleado. Se ha creído que la fortaleza metodológica es condición suficiente para dar cuenta de la fidelidad de los resultados (Picado y Ramírez, 1998: 13).

El privilegio a los métodos en la evaluación y en gran medida en las Ciencias Sociales, parte de suponer que los conocimientos se encuentran en el objeto evaluado o estudiado, luego el evaluador o el investigador debe circunscribirse exclusivamente en describir, acumular y sistematizar datos, de lo que es el objeto (Castells y de Ipola, 1983). En esa perspectiva, el método además de sistematizar lo evaluado, es el medio a través del cual se colectan datos. El empirismo y la evaluación necesitan de la descripción del objeto indagado, creen que solo con el registro, acumulación e interrelación de los datos seleccionados es posible revelar lo estudiado o evaluado, por ello privilegian al método de recolección de datos, así como, su procesamiento y presentación de los mismos.

El empirismo en la evaluación es continuador de una vieja tradición que atribuye al método como el fundamento de toda investigación, que es compatible con las creencias de una evaluación libre de valores, así como del objetivismo y el mecanicismo, porque se asume que las cantidades revelan la naturaleza de los objetos evaluados. Así, los objetos serán definidos según los datos escogidos y ponderados por los recolectores. Aparentemente, no existe teoría en el empirismo para evitar distorsiones y subjetividades supuestamente, aún cuando existen criterios ordenadores de los indicadores a ser colectados, construidos y procesados. El método, tanto para el empirismo y la evaluación, es lo más importante independientemente de la visión del sujeto evaluador, de la naturaleza y las circunstancias de lo evaluado, así como, las teorías e interpretaciones preliminares sobre lo evaluado. Sin embargo, el método tiene una determinación dependiente del objeto, sujeto y las teorías, en un contexto social e histórico particular; de ninguna manera puede ser independiente. El empirismo en la evaluación es una regresión al siglo XVII y XVIII, aún cuando ha estado presente en todos los tiempos.

Las ponderaciones sobre el método suelen ser etéreas, como cuando se discute cual es el mejor método, el cuantitativo o el cualitativo; lo cual es una discusión baldía, puesto que no hay cantidad sin cualidad, aún cuando pueden existir fenómenos que son inéditos y 
únicos. Sin embargo, es un razonamiento que ha dividido evaluaciones $y$ evaluadores e impide la actualización epistemológica (Cook y Reichardt, 2005).

El imaginar que los datos son pruebas incuestionables y objetivas de lo que se evalúa, además pueden ser interrelacionados e interpretados, independiente de cualquier postura teórica o ideológica, es un empirismo ingenuo (Chalmers, 1982). Sin embargo, la ponderación de los datos en la evaluación empirista adopta otras posturas contradictorias, se les otorga significación en razón de su contraste con modelos evaluativos, normas, estándares o criterios privados, lo cual es una contradicción empirista, pues esos registros ya no tendrían significación propia o en sí mismos, como regularmente se afirma, sino en relación con otros datos establecidos arbitrariamente. Así, se evalúa un proyecto o programa, con criterios que no emanan de la especificidad de las intervenciones sino en relación con modelos ideales o de otras realidades, lo cual evidencia la parcialidad de la evaluación y revela su propia inconsistencia, al negar que la verdad empirista se encuentre en el objeto.

La aparente validación intersubjetiva de las evaluaciones, no es por las evaluaciones mismas, sino por los modelos o criterios con los cuales se contrastan los resultados de ellas. El empirismo evaluativo se metamorfosea en formalismo, ahora la verdad está en el modelo o criterio evaluativo, por ello se afirma, relativizando el empirismo, que los datos son "crudos" o "brutos" (House y Howe, 2001). Sin embargo, pretender interpretarlos, según criterios predeterminados, es una contradicción empirista $y$ formalista, pues los datos tienen significación desde el mismo momento que fueron seleccionados, recolectados y procesados.

Dentro del concepto científico [...] se echa mano de métodos empíricos para obtención de la información y de procedimientos sistemáticos para su valoración con base en criterios a la vista, que posibilitan una verificación intersubjetiva (Stockmann, 2009: 68).
Su objetivo es generar información para que pueda usarse en la planificación y en la ejecución de programas y proyectos de desarrollo social (Picado, 2002a: 47).

La evaluación no solamente se refiere a un actuar específico, que tiene por objetivo tanto la obtención de información como la evaluación, sino que también se refiere al resultado de ese proceso (Stockmann, 2009: 68).

Las referencias de los evaluadores y teóricos de la evaluación ponen de manifiesto, la impertinencia de la evaluación positivista, por asumir que sus resultados tienen verificación intersubjetiva, quizás por usar criterios ajenos para validar los propios o porque las cantidades son interpretadas de manera idéntica; porque cree que sus datos pueden ser usados para fines que no se relacionan con los datos ni las realidades evaluadas como la planificación, etc. y porque se pueden controlar los resultados de la evaluación. Asimismo, los evaluadores y los profesionales de la estadística, usan los datos para proyectarlos, lo cual violenta la significación de los mismos, pues en todos los casos, están circunscritos al tiempo-espacio que fueron registrados, sin significación ni validez para otros contextos ni circunstancias.

De ahí que los evaluadores deban asumir la función de facilitadores neutrales en cuanto a su forma de manejar los datos y los valores de las personas. Desde este punto de vista, los conocimientos y destrezas no desempeñan ningún papel especial. Las personas deben decidir por su cuenta. Todo es relativo a la concepción del individuo (House y Howe, 2001: 19).

Al parecer la trasmutación empirista, formalista e individualismo metodológico en la evaluación, no tienen ninguna regla establecida, se adopta cualquiera, dependiendo del criterio de los evaluadores y los usos indistintos de los clientes de los evaluadores, lo cual revela un precario compromiso ético y profesional. 
Se podría afirmar que independientemente de la metamorfosis epistemológica $y$ metodológica de la evaluación, a los datos se les otorga funciones legitimadoras e incontrovertibles. Así, muchas evaluaciones no son más que puros datos para cualquier interpretación u aplicación. De este modo, los datos serían la expresión última y definitoria de justificaciones racionalistas, tecnocráticas e instrumentalistas, aún cuando al pragmatismo evaluativo, poco le importa la consistencia epistemológica.

\section{LA EVALUACIÓN Y EL PODER}

La discusión sobre concepción en la evaluación y sus implícitos, no son solo de carácter epistemológico, reviste también una posición autoritaria y omnipotente al convertir a la realidad en objeto de decisiones privadas; la de asumir el control del futuro o el supuesto de tener la verdad absoluta más allá del quehacer científico. Aspectos que ponen de manifiesto una valoración cultural e ideológica arraigada en occidente $y$ fortalecida por las relaciones capitalistas. Ello no implica solamente una cosmovisión mecanicista del mundo, también supone el arrogarse autoridad para incidir intencional y premeditadamente sobre el devenir social de organizaciones y naciones. La pluralidad de las relaciones sociales $y$ la heterogeneidad de contextos no son consideradas importantes, solo importa las decisiones y acciones sobre las realidades, sin duda podría parecer irrelevante refutar tales prácticas por inoperantes y absurdas, pero los procesos autoritarios de las prácticas empresariales y estatales, sin duda afectan la vida $y$ condiciones de quienes son víctimas de esas actuaciones. La facultad privada para decidir y actuar no es garantía para la obtención de premeditados resultados, ni construye organizaciones democráticas, pero los poderes desiguales en la sociedad contribuyen a privatizar beneficios y socializar las crisis privadas.

La evaluación concebida como el medio para verificar los aciertos o errores de las intervenciones para la consecución de determinados propósitos, es también el paso previo para nuevas intervenciones. En tal sentido, la evaluación también podría ser apreciada como un recurso y una justificación que se efectúa desde el poder para el poder (Huaylupo, 1995) y desde luego, es importante para quienes disponen del trabajo de otros, así como, de la propiedad sobre los recursos para el diseño, programación y ejecución de las acciones en ámbitos sociales, ambientales o naturales.

Los espacios de intervención de las organizaciones privadas, estatales o internacionales, es también el espacio de actuación de las evaluaciones. El universo de la labor evaluativa está pautado y delimitado por el poder decisorio y en tal sentido, forma parte del quehacer administrativo de las organizaciones $y$ de las consideraciones tecnocráticas que predominan en su quehacer, así como, guarda similitudes en su relación supeditada al poder en las entidades orgánicas (Huaylupo, 2002).

La evaluación trabaja, se identifica y propone en razón del poder en la organización, pero como todo ente orgánico, su existencia está dada para interactuar sobre la sociedad y organizaciones, como condición para la reproducción y expansión organizacional en la sociedad. Una evaluación que contemple las acciones en la organización o los criterios privados, no solo será omisa de comprender los resultados obtenidos, así como, serán fallidas las recomendaciones de acción correctiva, dada la ignorancia sobre las aproximadas determinaciones que condicionan los resultados en las organizaciones, también será responsable solidaria de decisiones $y$ acciones privadas que atentan contra la democracia y la pluralidad social.

La manipulación de la realidad es una posición fundada por una praxis del poder, la cual se encubre de técnica. La evaluación como manifestación del poder, pone en evidencia las inconsistencias y contradicciones con los argumentos dados sobre la objetividad, neutralidad y racionalidad de su quehacer.

En la actualidad, la evaluación está articulada con las propuestas de "rendición de cuentas" o la transparencia de las intervenciones estatales en los programas sociales $y$ proyectos de inversión. La evaluación privada o de proyectos estatales no relativiza lo discutido, por el contrario podría ser confirmado, en un contexto donde los estados que han perdido capacidad reguladora y planificadora de la 
sociedad, o dicho de otro modo, en el liberalismo contemporáneo estas sus acciones estatales son desarticuladas de manera institucional y sectorialmente, sin obedecer a las necesidades, problemáticas ni aspiraciones sociales. En este contexto, en donde predomina la trasferencia privada de la obra y función pública, la evaluación es similar a las efectuadas de manera privada, ya que son justificadoras de quehaceres y poderes autocráticos, los cuales están preocupados solo por la rentabilidad y el crecimiento económico.

La necesidad de la evaluación requiere ser redefinida epistemológica, teórica, política y éticamente. Destacar una actuación evaluativa en torno del poder de propietarios y del Estado, ha constituido una justificación a los intereses que privatizan el bienestar y el crecimiento. El quehacer del evaluador, en este contexto social y político, privatiza también su desempeño profesional.

Los evaluadores no deben ignorar los desequilibrios de poder ni suponer que el diálogo sobre la evaluación sea abierto cuando no lo sea. Hacerlo significa aceptar de forma implícita el statu quo de las estructuras de poder. Creemos que la mejor solución para los evaluadores consiste en afrontar directamente las cuestiones de poder $y$ adoptar una postura de deliberación democrática como ideal para juzgar las afirmaciones públicas de valor. En esta concepción, los evaluadores no son espectadores ni reyes filósofos (House y Howe, 2001: 26).

El contexto no es indiferente al quehacer $y$ pensamiento científico, como tampoco puede serlo para la evaluación. Las justificaciones técnicas $y$ políticas que validan $y$ privilegian el interés privado, impuesto como interés general en la sociedad, sin duda merece el debate $y$ la crítica en el espacio académico de la institucionalidad pública y social, porque compromete el desarrollo, el bienestar social y la libertad.

\section{BIBLIOGRAFÍA CITADA}

Amezcua, Cesáreo. "Introducción a la evaluación”. En: Amezcua, Cesáreo y Jiménez, Antonio. Evaluación de programas sociales. España: Ediciones Díaz de Santos, 1996.

Backhoff, Eduardo; Tirado, Felipe y Larrazolo, Norma. "Ponderación diferencial de reactivos para mejorar la validez de una prueba de ingreso a la universidad". Revista Electrónica de Investigación Educativa 3 (1). Universidad Autónoma de Baja California, 2001.

Bunge, Mario. La ciencia. Su método y filosofía. Buenos Aires: Editorial Sudamericana, 1997.

Carr, Edward. ¿Qué es la historia? Barcelona: Editorial Seix Barral, 1978.

Castells, Manuel y de Ipola, Emilio. "Epistemología y Ciencias Sociales". Cuadernos Teoría y Sociedad. México: Universidad Autónoma Metropolitana Azcapozalco, 1983.

Chalmers, Alan. ¿Qué es esa cosa llamada ciencia? Una valoración de la naturaleza y el estatuto de la ciencia y sus métodos. España: Editorial Siglo Veintiuno, 1982.

Cohen, Morris R. "Introducción a la Lógica". Breviarios 67. México: Editorial Fondo de Cultura Económica, 1965.

Cook, Thomas y Reichardt, Charles. Métodos cualitativos y cuantitativos en la investigación evaluativa. Madrid: Editorial Morata, 2005.

De Paula, Francisco. "¿Qué nos dicen los indicadores económicos?”. Revista INCAE 6 (2). Instituto Centroamericano de Administración de Empresas, 1992: 9-23.

Epicteto. Manual de Epicteto. Argentina: Biblioteca Nueva Era, 2003. En: $<w w w . p r o m i n e o . g q . n u>$

Escudero, Tomas. "Claves indentificativas de la investigación evaluativa: análisis desde la práctica". Revista Contextos Educativos 8-9. España, 2006: 179-199.

Garcia, Roberto. "Utilización de indicadores predictivos: ¿pensamiento científico o mágico?". Revista Médica de Rosario 7. Argentina, 2006: 111-116.

García de la Huerta, Marcos. "Nacionalismo y filosofía”. Revista Diálogos 83. Año XxxviII. Puerto Rico. Departamento de 
Filosofía de la Universidad de Puerto Rico, 2003: 191-204.

González, Francisco. Economía para gerentes $y$ administradores. Ecuador: INCAE, 1995.

Gouldner, Alvin. Sociología actual: renovación y crítica. Madrid: Alianza Editorial, 1979.

Gramsci, Antonio. Cuadernos de la cárcel. Tomo 4. Velantino Gerratana (coord.). Edición crítica del Instituto Gramsci. México: Ediciones Era, 1986.

Habermas, Jürgen. Ciencia y técnica como 'ideología'. Madrid: Editorial Tecnos, 1984.

Horkheimer, Max. Crítica de la razón instrumental. Buenos Aires: Editorial Sur, 1973.

House, Ernest. Evaluación, ética y poder. Madrid: Ediciones Morata, 1994.

House, Ernest y Howe, Kenneth. Valores en evaluación e investigación social. Madrid: Ediciones Morata, 2001.

Huaylupo, Juan. "La relatividad y significación de los datos". Cinta de Moebio. Revista de Epistemología de Ciencias Sociales 32. Santiago de Chile. Facultad de Ciencias Sociales. Universidad de Chile, 2008: 127152.

Huaylupo, Juan. "La administración: una determinación dependiente de las relaciones sociales". Revista de Administración Pública: Ética y Gerencia Pública 33-34. San Juan de Puerto Rico: Universidad de Puerto Rico. Escuela Graduada de Administración PúblicaRecinto Río Piedras, 2002: 117-132.

Huaylupo, Juan. "La evaluación del desempeño ¿un enfoque individual del trabajo colectivo o una visión individualista del trabajo social?". Revista Fiscalización y Gestión Pública 2 (2). San José. Contraloría General de la República y Universidad de Costa Rica, 1995: 73-84.

Luhmann, Nikla. Sistemas Sociales. Barcelona: Editorial Anthropos-Universidad Iberoamericana de México-Pontificia Universidad Javeriana de Colombia, 1998. Maturana, Humberto y Varela, Francisco. El árbol del conocimiento. Barcelona: Editorial Debatep, 1999.
Montero, Eiliana. "Educación e ingreso como predictores de la esperanza de vida: evidencias de un análisis de regresión aplicado a indicadores de desarrollo humano". Revista Ciencias Sociales 94. Costa Rica. Universidad de Costa Rica, 2001: 71-60.

Montero, Eliana. "Marco conceptual para la evaluación de programas en salud". Población y Salud en Mesoamérica 1 (2). San José: Centro Centroamericano de Población. Universidad de Costa Rica, 2004. [Revista Electrónica]. En: <http:// ccp.ucr.ac.cr>

Montero, Eiliana; Villalobos, Jeannette y Valverde, Astrid. "Factores institucionales, pedagógicos, psicosociales y sociodemográficos asociados al rendimiento académico en la Universidad de Costa Rica: un análisis multinivel". Revista Electrónica de Investigación y Evaluación Educativa (RELIEVE) 13 (2). España: Universidad de Valencia, 2007: 215-234.

Morin, Edgar. Autobiografía de Edgar Morin. 1995. En: <www.pensamientocomplejo. com.ar> [consultado en julio de 2011].

Neffa, Julio. El proceso de trabajo y la economía de tiempo. Argentina: Editorial Humanitas $y$ Centre de Recherche et Domentation sur L’Amerique Latine, 1990.

Parsons, Talcott. La Estructura de la Acción Social. Madrid: Ediciones Guadarrama, 1968.

Picado, Marta. “Cómo podría delinearse una evaluación cualitativa?". Revista Ciencias Sociales 97 (III). Costa Rica: Universidad de Costa Rica. 2002: 47-61.

Picado, Xinia. "Criterios para realizar evaluaciones de calidad." Revista Ciencias Sociales 97 (III). Costa Rica: Universidad de Costa Rica, 2002: 9-16.

Picado, Xinia y Ramírez, Edgar. "Razonamiento evaluativo". Revista Reflexiones 97. Costa Rica. Universidad de Costa Rica, 1998: 13-29.

Prigogine, Ilya. El fin de las certidumbres. Santiago de Chile: Editorial Andrés Bello, 1996. 
Stocmann, Reinhard. Evaluación y desarrollo de la calidad. Costa Rica: Editorial Universidad de Costa Rica, 2009.

Weber, Max. La ética protestante y el espíritu del capitalismo. Barcelona: Ediciones Península, 1977.

Weber, Max. Ensayos sobre metodología sociológica. Argentina: Amorrortu editores, 2001.
Zemelman, Hugo. "La relación de conocimiento y el problema de la objetividad de los datos". Estudios Sociológicos 33. México. Colegio de México, 1993: 641-659.

Fecha de ingreso: 12/10/2010 Fecha de aprobación: 11/08/2011 